\title{
Rol del farmaceutico comunitario en la identificacion De factores de riesgo cardiovascular en la menopausia
}

\section{Role of the community pharmacist in the identification of Of cardiovascular risk factors during menopause}

DOI: $10.46932 / \mathrm{sfjdv} 2 \mathrm{n} 4-037$

Received in: May 1st, 2021

Accepted in: Jun 30th, 2021

\section{Claudia E. Guerrero}

Médica. Especialista en Tocoginecología. Especialista en Farmacología Clínica. Doctora en Medicina y Cirugía. Docente universitaria UNC. IUCBC. UNVM. UCC.

\section{María E Ruggieri}

Estudiante. Facultad de Medicina. IUCBC.

\section{Matías Pernochi Scerbo}

Estudiante. Facultad de Ciencias Químicas. UNC

María Mercedes Rencoret

Farmacéutica. Directora. Portal Educativo Consenso Salud

\section{INTRODUCCIÓN}

En la actualidad, la esperanza de vida se ha incrementado en todo el mundo hasta la octava década. (Lobo RA, 2018; Ahuja M, 2016)

Las enfermedades cardiovasculares (ECV) siguen siendo la principal causa de muerte en muchos países en vías de desarrollo, pero también en países desarrollados. (Bolaños Chavez, BD; 2021) y representa una de las principales causas de mortalidad en mujeres postmenopáusicas. (Arteaga Urzúa E; 2016).

La menopausia natural se define como la ausencia de menstruación durante un período de doce meses, la cual ocurre generalmente entre los 49 y 52 años. Un 5\% de las mujeres desarrolla una menopausia precoz (entre los 40/45 años) y aproximadamente un 1\% presenta una menopausia prematura (antes de los 40 años) también conocida como insuficiencia ovárica primaria. (Santoro N, 2015; Benvegnu, C, 2019) Posterior a la menopausia, se presentan múltiples factores que aumentan el riesgo real de enfermedad cardiovascular, dentro de los cuales se incluye el tabaquismo, la hipertensión arterial, las dislipidemias, la diabetes mellitus tipo dos, la edad de la menarca y de la menopausia, así como también los síntomas vasomotores, además de los factores de riesgo más comunes del período de transición, tales como la disminución de la tolerancia a la glucosa y la disfunción endotelial. (Abernethy K. Managing,2016) 
Las ECV se constituyen como un factor predisponente importante de discapacidad, que impacta de manera considerable sobre la calidad de vida de las personas, además del elevado gasto sanitario que suponen debido a su alta prevalencia. (Zhu D, 2019; Young L, 2019)

La OMS asegura que la mayoría de las ECV se pueden prevenir actuando sobre los factores de riesgo modificables. (OMS. 2019).

La farmacia comunitaria, es uno de los eslabones de fundamental importancia en el sistema de salud, en donde el profesional farmacéutico adquiere un rol preponderante para el seguimiento de la farmacoterapia de los pacientes; el acceso directo y personalizado en la consulta sobre posibles reacciones adversas de medicamentos indicados para el tratamiento de las ECV, la colaboración para favorecer la adherencia de los mismos favorecen su rol como agente sanitario en la prevención; elementos claves para alcanzar los objetivos y cumplir con las pautas sanitarias dentro del equipo de salud.

Existen varios paradigmas sobre la relación profesional de la salud - paciente: paternalista o hipocrático; tecnológico; legal; comercial o consumista; de alianza. Todo profesional deberá encontrarse dentro de alguno o varios de estos modelos. Dependiendo del contexto puede variar el accionar del facultativo, pero el profesional es el primer y último responsable de los modos y terapéutica a asumir. Por esta razón es primordial el reflexionar sobre el pensar y el hacer profesional. La relación profesional de la salud - paciente puede ser lo que determine el éxito o el fracaso del tratamiento y pronóstico (Risk K; 2021)

\section{OBJETIVOS}

- Identificar factores de riesgo cardiovascular en la población de mujeres en climaterio/menopausia que acuden a la farmacia comunitaria, en el período comprendido desde el 1 de octubre al 1 de noviembre de 2020 en Argentina.

- Colaborar para que el profesional farmacéutico brinde en forma integral atención a la salud de la mujer en la etapa del climaterio.

\section{MATERIALES Y MÉTODO}

La investigación se desarrolló mediante un enfoque cuantitativo, se planteó como un estudio de corte transversal, observacional, realizado en oficinas de farmacia comunitaria, en el período comprendido de octubre a noviembre de 2020. El instrumento utilizado fue una encuesta validada. (Matzumura-Kasano J, 2020)

Las farmacias involucradas correspondían distintas provincias de la Argentina. 
Se encuestaron 576 mujeres menopaúsicas que acudieron a la oficina de farmacia en forma espontánea, con el objetivo de identificar factores de riesgo cardiovascular.

Las pacientes que accedían a la consulta, respondieron una encuesta donde se destacaban factores de riesgo cardiovascular relacionados a este período de la vida: cigarrillo, hipertensión arterial, dislipemias, diabetes.

Integraron este estudio 65 profesionales farmacéuticos, un profesional médico y dos estudiantes (farmacia y medicina) entrenados previamente en la detección de factores de riesgo cardiovascular y su implicancia en el desarrollo de enfermedad cardiovascular (ECV) en la menopausia.

Tamaño muestral: estudio piloto, se establece entre 8 y 10 mujeres por farmacia.

Variable principal: Edad, fecha de última menstruación, hábitos tóxicos: cigarrillo.

\section{Procedimiento:}

- Ofrecimiento del estudio.

- Realización del cuestionario sobre factores de riesgo cardiovascular: HTA, dislipemia, sedentarismo, diabetes y/o resistencia la a la insulina, tratamientos farmacológicos

- Intervención del farmacéutico: en función del resultado, el farmacéutico comunitario realizará la intervención oportuna: educación sanitaria, indicación farmacéutica o derivación a atención primaria.

\section{RESULTADOS}

El análisis de las respuestas arrojó los siguientes resultados:

La edad promedio de las participantes en el estudio fue de 60 años, (45 a 86 años). La encuesta fue realizada al azar, en oficinas de farmacia comunitaria. No implicó el reclutamiento de este grupo etario.

La edad promedio de fecha de última menstruación (FUM) fue de 53 años, pero la mayoría de las mujeres encuestadas refieren que su FUM fue a los 50 años.

Reconocían entre los hábitos tóxicos asociados al riesgo de enfermedad cardiovascular el hábito tabáquico, así mismo el $20 \%$ de las encuestadas eran fumadoras activas.

En relación a quienes presentaban diagnóstico de hipertensión arterial, un 31\% de las encuestadas estaban medicadas con antagonistas de la enzima convertidora: $15 \%$ con IECA especialmente enalapril y un $16 \%$ con ARA 2. Otras terapias implementadas fueron: $9 \%$ antagonistas cálcicos; 1,7 con bloqueantes beta (BB) y 9\% con combinaciones de fármacos.

Para el tratamiento de las dislipemias existe un mercado muy amplio de drogas hipolipemiantes. La utilización de una u otra depende del riesgo cardiovascular del paciente y de la fracción de lípidos que 
se desee modificar. Las dislipemias fueron un factor de riesgo identificado en el $39 \%$ de quienes participaron en el estudio, ya que contaban con diagnóstico y estaban en tratamiento: $28 \%$ con estatinas, $0,7 \%$ con fibratos, $2,2 \%$ con combinación de fármacos hipolipemiantes y 1,2\% ezetimibe.

Las pacientes incluidas en la serie manifestaron cambios en la imagen corporal, lo relacionaron con el aumento ponderal ya que $22 \%$ eran obesas y $41 \%$ presentaban sobrepeso; De total de la serie estudiada, un $9 \%$ se encontraban en tratamiento con metformina, $2 \%$ con asociaciones de hipoglucemiantes y $0,9 \%$ con insulina.
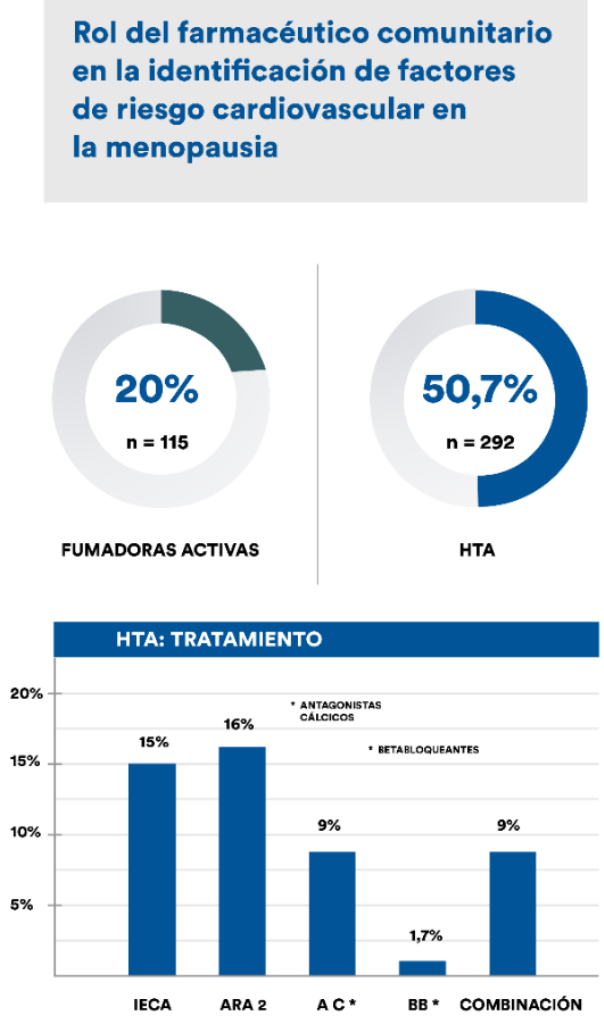

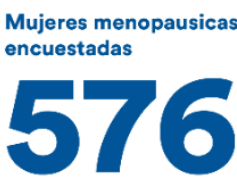

(45 y 86 años)

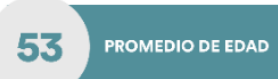

50

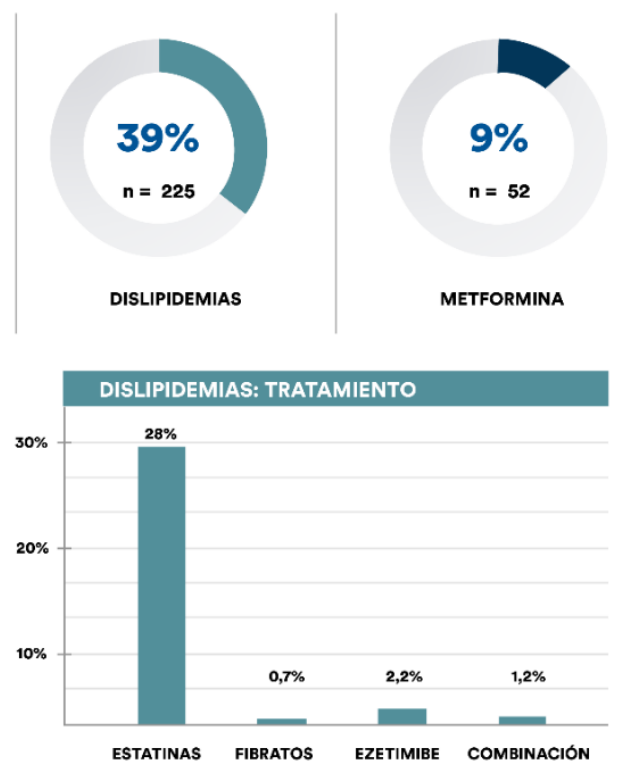

\section{DISCUSIÓN}

La Práctica Farmacéutica necesita investigar en gestión, no sólo de la farmacia como empresa, sino de la integración de los nuevos servicios cognitivos en la organización que es. Necesita investigar en nuevas habilidades para proveer nuevos servicios; explorar las actitudes de los actuales profesionales y de los estudiantes de farmacia, su relación con otros profesionales de la salud y sobre todo, con los pacientes y con los médicos, sobre habilidades de comunicación. Es importante también evaluar los servicios cognitivos existentes en la actualidad incluidos sus resultados clínicos, humanísticos y económicos. (Gastelurrutia Garralda, MA; 2010) 
Las mujeres tienen mayor percepción del problema de salud. (Mosca L, et al. 2000) y la menopausia es a veces un problema mal entendido y poco consultado por las mujeres que al mismo tiempo demandan soluciones. Las acciones de educación sanitaria en una oficina de farmacia deben ser realizadas centrándose en las demandas de las pacientes. (Pérez Benajas MA, et al. 2010)

La transición que experimenta la mujer durante la menopausia se asocia con el desarrollo de características propias del síndrome metabólico (SM), entre las que se encuentran: aumento de la grasa central abdominal, alteración del perfil lipídico y resistencia a la insulina. (Monsalve, C, 2017). Por esta razón, la prevalencia de SM se incrementa con la menopausia hasta en un 60\% (Estepa Galindo, E, 2020). La verdadera importancia de los factores que influyen en el riesgo cardiovascular en las mujeres posmenopáusicas está lejos de determinarse con exactitud y total certeza; estas pacientes deberían ser tratadas en forma temprana e integral con el fin de reducir su mortalidad. (Rojas S, 2014; Maas A, 2021)

La enfermedad cardiovascular es la primera causa de morbimortalidad en la mujer, situación que es modulada por los procesos de desarrollo ontogenético, especialmente los relacionados con la transición menopáusica. (Estepa Galindo, E, 2020). Se presentan múltiples factores que aumentan el riesgo real de enfermedad cardiovascular en mujeres postmenopáusicas, entre ellos se concluyen los siguientes: el tabaquismo, la hipertensión arterial, las dislipidemias, la diabetes mellitus tipo dos, la edad de la menarca y de la menopausia, y los síntomas vasomotores, y factores de riesgo tradicionales del período de transición, tales como la disminución de la tolerancia a la glucosa, el aumento de la presión arterial y la disfunción endotelial. (Martorell M, et al; 2020)

\section{CONCLUSIONES}

Concluimos que la red de farmacias, se posicionan como establecimientos sanitarios estratégicos en la prevención del riesgo cardiovascular entre las mujeres en menopausia que acuden a ellas.

La pandemia por COVID19 disminuyó las consultas en hospitales y centros de salud y en este sentido, destacamos también, que las claves que posicionaron a la farmacia comunitaria son la accesibilidad, la distribución estratégica y cercanía, y la comunicación farmacéutico/paciente que permite brindar el asesoramiento adecuado en tiempo y espacio, evaluar la fiabilidad de la información y prevenir factores de riesgo cardiovascular.

La participación activa de los profesionales farmacéuticos en una red de salud distribuida estratégicamente con alcance a la población en general, garantiza la atención integral de cada paciente, siendo un eslabón fundamental para la concreción de resultados que mejoren la calidad de vida, en este caso de mujeres durante la menopausia. 


\section{BIBLIOGRAFIA}

1. Lobo RA. Menopause and aging. En: Strauss J, Babieri R, editores. Yen and Jaffe's Reproductive Endocrinology. 8a ed. Filadelfia: Elsevier Inc.; 2018: 308-339.e8 2.

2. Ahuja M. Age of menopause and determinants of menopause age: A PAN India survey by IMS. J Midlife Health. 2016; 7(3): 126-131. DOI: 10.4103/0976-7800.191012

3. Bolaños Chavez, BD y Ortega Muñoz; E. Riesgo cardiovascular asociado a menopausia. Revista Médica Sinergia Vol.6(1), 2021-ISSN:2215-4523 / e-ISSN:2215-5279

a. Disponible en: http://revistamedicasinergia.com

4. Santoro N, Epperson CN, Mathews SB. Menopausal symptoms and their management. Endocrinol Metab Clin North Am. 2015; 44(3): 497-515. DOI: 10.1016/j.ecl.2015.05.001

5. $\quad$ Benvegnu, C; Paz, V; Singh, L; Torres, F; Otero, P y Kozak A. Insuciencia Ovárica Prematura. Rev Argent Endocrinol Metab. 2019; 56 \#1

6. Abernethy K. Managing short-term menopausal symptoms. En: NICE guideline: Menopause: diagnosis and management. Post Reprod Health. 2016; 22(2): 63-66. DOI: 10.1177/2053369116648269

7. Zhu D, Chung HF, Dobson AJ, Pandeya N, Giles natural menopause and risk of incidentcardiovascular disease: individual patient data. Lancet Public Health 2019; 4: e553-64

GG, Bruinsma F, et al. Age at a pooled analysis of

a. Disponible en: https://doi.org/10.1016/S2468-2667(19)30155

8. Young L, Cho L. Unique cardiovascular risk factors in womenHeart 2019;105:1656-1660.

9. OMS. Prevención de las enfermedades cardiovasculares. Guía de bolsillo para la estimación y el manejo del riesgo cardiovascular. 2019

10. Risk K; Guerrero C; Molina J; Pelliccioni P. (2021). Relación profesional de la salud paciente. South Florida Journal of Development, Miami, v.2, n.3, p. 3956-3962 special edition, jul. 2021. ISSN

11. Matzumura-Kasano J; Gutierrez Crespo HJ; Wong-Mac JC; Alamo-Palomino IJ. Instrumento para la evaluación del climaterio y la menopausia en el primer nivel de atención. Rev. Fac. Med. Hum. Octubre 2020;20(4):560-567. DOI 10.25176/RFMH.v20i4.3057

12. Arteaga Urzúa E. Menopausia y riesgo cardiovascular. Rev Med Chil. 2016 2016;144( 11):1375-1376.

a. Disponible en: http://dx.doi.org/10.4067/S0034-988720160011000015.

13. Gastelurrutia Garralda MA. Investigación en farmacia comunitaria. FARMACÉUTICOS COMUNITARIOS 2010; 2(1): 3

14. Mosca L, Jones WK, King KB, Ouyang P, Redberg RF, Hill MN. Awareness, perception, and knowledge of heart disease risk and prevention among women in the U nitedStatesAmerican Heart Association Women'sHeart Disease and Stroke Campaign TaskForce. Arch Fam Med. 2000 Jun;9(6):506-15. doi: 10.1001/archfami.9.6.506. PMID: 10862212. 
15. Pérez Benajas MA, Vázquez Medem M, Juan Honrrubia J, Álvarez Sánchez E, Valle Carcelén E. La menopausia, época de cambio. Soluciones desde la farmacia comunitaria. FARMACÉUTICOS COMUNITARIOS 2010; 2(1): 10-14

16. Monsalve, C, Reyes, V., Parra, J. Blümel. Orientaciones clínicas para el manejo de la menopausia. REVCOG 2017; 22:(1):14-20

17. Estepa Galindo, E; Prado Martínez, C; Carmenate Moreno, M; Acevedo Cantero, P; García García, C. de J \& Marrodán Serrano, M. D. (2020). Edad de menopausia, condición nutricional y componentes del síndrome metabólico en mujeres españolas. Nutrición Clínica Y Dietética Hospitalaria, 40(4).

a. Disponible en: https://doi.org/10.12873/404marrodan

18. Rojas S; Lopera V JS; Cardona VJ; Vargas NG; Hormaza MP. Rev. chil. obstet. ginecol. vol.79 no.2 Santiago 2014

a. Disponible en: http://dx.doi.org/10.4067/S0717-75262014000200010

19. Maas A; Rosano G; Cifkova R; Chieffo A; van Dijken D; Hamoda H; Kunadian V; Laan E; Lambrinoudaki I; Maclaran K; Panay N; Stevenson J; van Trotsenburg M and Collins C. Cardiovascular health after menopause transition, pregnancy disorders, and other gynaecologic conditions: a consensus document from European cardiologists, gynaecologists, and endocrinologists. European Heart Journal (2021) 00, 1-18 ESC REPORT

a. Disponible en http://dx.doi:10.1093/eurheartj/ehaa1044

20. Martorell $\mathrm{M}$ et al. Menopausia y factores de riesgo cardiovascular en mujeres chilenas.

Rev. Med Chile. 2020; 148:178-186 